\title{
X-Ray Mapping Using a Multiple-EDS (DUAL) Detectors
}

\author{
R. Wuhrer ${ }^{1}$, K. Moran ${ }^{1,2}$, M. R. Phillips ${ }^{1}$ and P. Davey ${ }^{2}$ \\ ${ }^{1}$ Microstructural Analysis Unit, University of Technology, Sydney, PO Box 123 Broadway, \\ Australia. \\ ${ }^{2}$ Moran Scientific Pty Ltd, P.O. Box 651, Goulburn, NSW, 2580 Australia.
}

X-ray mapping (XRM) is an extremely useful problem solving tool. However, the two major problems for energy dispersive spectroscopy are interpretation of results under non ideal conditions (strong overlap and small peak size relative to background), and the time required to obtain a good quality $256 \times 256$ pixel map ( 1 to 3 hours). XRM has been considered a slow technique, claiming many hours of SEM time and often relegated to out-of-hours (overnight) mapping. With the development of high count rate silicon high resolution drift detectors (SDD) $[1,2]$ and multi-detector systems [3], the time required to acquire XRM decreases. With a single EDS detector at 20kcps output, a good 512x512 quantitative map can be obtained in around 4 to 8 hours for major elements ( $>10 \mathrm{wt} \%$ evenly distributed) and minor elements ( $>1 \mathrm{wt} \%$ localised).

With the development of the SDDs, X-ray mapping at count rates of 100kcps to 1000kcps output are possible, thus allowing $256 \times 256 \mathrm{X}$-ray maps in around 3 to 15 minutes, depending on the count rate. The accuracy of the EDS results and the quality of the X-ray maps depends on the elements present in the sample. If there are no overlaps between elements in your sample, then these higher count rate detectors can be used to map qualitatively. For quantitative work, it is still not desirable to use these detectors at the higher count rate, as the spectra are of poor resolution (190 to $250 \mathrm{eV}$ ) with other artefacts.

It is now possible to quantitatively map using multi-detectors and combined detectors such as WDSEDS, hence not only improving count rate capability but also improving the ability to map trace elements very accurately [3]. Mapping with multiple detectors enables better quality maps to be obtained through use of lower beam currents, thus improving the map quality as well as minimizing other problems such as beam drift. The combination of new detectors and multi-detector systems now opens up new possibilities in high speed X-ray mapping. Using a single detector at higher count rates can lead to large beam currents, higher pulse pile up, loss of energy resolution, gain and zero drift, higher dead times, specimen damage, specimen charging and poorer image resolution.

The introduction of a second EDS X-ray detector can reduce the map time by half, for example from 2 hours to 1 hours. This is now becoming a more acceptable time frame for X-ray mapping, but still is too long. It is important to realise that each time you double the number of detectors you halve the time to collect a map (the biggest time advantage is in adding a second detector). However, a two time statistical advantage requires four times the number of detectors.

A more acceptable time frame would require at least ten EDS or other X-ray detectors thus reducing the time ten fold. Therefore, the $512 \times 512$ EDS map collected at $20 \mathrm{kcps}$ could be reduced to less than sixty minutes. To obtain high quality spectra we must limit our performance to that range where the spectra does not suffer from any adverse distortion. This range is around 20kcps and detector resolution of below $150 \mathrm{eV}$. So the best way to map is by the use of a multi-detector array (with at 
least ten detectors) where the total combined x-ray count rate is of the order of 200kcps with $20 \mathrm{kcps} /$ detector operating at high resolution and low pulse pile-up.

After mapping with multiple detectors, it is very hard to go back to mapping with a single detector. It is a bit like asking someone to analyse for twice, and even four times, as long as they currently do. For an increase in map quality (statistics), the magic number of detectors required is the square root of the number of detectors. Whereas calculating the new analysis time, is the current analysis time divided by the number of detectors. Therefore, if current practice is to analyse a sample for 100 seconds, then through using four detectors the analysis time will only need to be 25 seconds for the same result. However, if you do count for 100 seconds with four detectors, the detection limit can be halved and precision doubled.

So why are we not all using multiple detectors? Previously it has been very expensive to add more than one detector, but with present cost trends it is a very cost effective solution to tying up machine time with long x-ray maps. Furthermore, there are currently only a few manufacturers who provide software for multiple detectors. The most effective time reducer is the adding of one extra detector. This can be done easily by putting another detector onto the column. Two separate detectors can be brought in very close to the sample, thus increasing detection efficiency, but this requires an extra dewar to fill. This can also help with analysis of rough samples. However some instruments do not allow detectors to be positioned close to the sample. In these cases it is preferable to have the X-ray detectors mounted within the single turret of the detector assembly, thus allowing both to be cooled by the same dewar.

A twin EDS detector in a single turret (dual detector) with only one dewar has been built and incorporated on a microscope so that we can evaluate it compared to two individual detectors. Initial problems such as electrical interference, temperature interference and poor collection efficiency due to mechanical placement behind the Be window have been overcome. The advantage of this type of system is that the spectra can be summed as all the detectors are very similar.

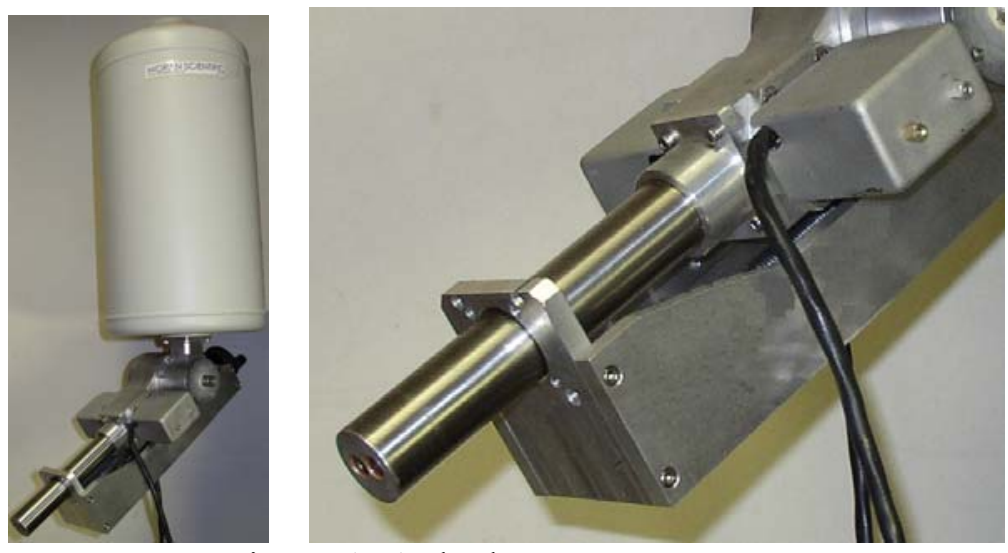

Figure 1: A dual Detector system.

\section{References}

[1] D. Newbury, Microsc.Microanal 11(Suppl 2) Proceedings (2005) 1328.

[2] T. Schulein, Microsc.Microanal 11(Suppl 2) Proceedings (2005) 460.

[3] R. Wuhrer, K. Moran, M. R. Phillips and P. Davey, Microsc.Microanal 11(Suppl 2) Proceedings (2005) 466. 